\title{
Resource Allocation for UAV Relay-Assisted IoT Communication Networks
}

\author{
Dinh-Hieu Tran, Van-Dinh Nguyen, Sumit Gautam, Symeon Chatzinotas, Thang X. Vu, Björn Ottersten \\ Interdisciplinary Centre for Security, Reliability and Trust (SnT), University of Luxembourg, Luxembourg. \\ Email: \{hieu.tran-dinh, dinh.nguyen, sumit.gautam, symeon.chatzinotas, thang.vu, bjorn.ottersten\}@uni.lu
}

\begin{abstract}
This work studies unmanned aerial vehicle (UAV) relay-assisted Internet of Things (IoT) communication networks in which a UAV is deployed as an aerial base station (BS) to collect time-constrained data from IoT devices and transfer information to a ground gateway $(\mathrm{GW})$. In this context, we jointly optimize the allocated bandwidth, transmission power, as well as the UAV trajectory to maximize the total system throughput while satisfying the user's latency requirement and the UAV's limited storage capacity. The formulated problem is strongly nonconvex which is very challenging to solve optimally. Towards an appealing solution, we first introduce new variables to convert the original problem into a computationally tractable form, and then develop an iterative algorithm for its solution by leveraging the inner approximation method. Numerical results are given to show significant performance improvement over benchmark schemes.
\end{abstract}

Index Terms-Information freshness, Internet of Things, timely data collection, unmanned aerial vehicle (UAV).

\section{INTRODUCTION}

Nowadays, IoT has gained significant penetration in many areas such as e-health, smart city, agriculture, and smart grid [1], [2]. The number of IoT devices is estimated up to 100 billions by 2025 [3]. Nevertheless, as the restriction of available resources for wireless communication systems, the design of efficient IoT communications is becoming a daunting challenge. More specifically, since base stations (BSs) are commonly installed in permanent locations with fixed antenna height as well as their sparse distribution which results in a degraded performance in the case of severe fading or overloaded scenarios. It is infeasible to solve these problems by simply increasing the number of BSs due to high installation and maintenance costs [4]. Therefore, conventional systems hardly satisfy the requirements of IoT networks. Moreover, in emergency circumstances such that BSs are destroyed/isolated after a disaster, they are unable to operate normally. Fortunately, UAV communications could become a promising technology to overcome the above drawbacks thanks to their mobility, agility, and flexible deployment [5]. More specifically, the UAV can fly closer to the IoT devices which significantly enhances the network performance and reduces the total power consumption of IoT users.

The timely data collection or information freshness in UAVassisted IoT communication networks has attracted considerable attention recently [6]-[9]. In emergency scenarios, outdated data may cause untrustworthy controllable decisions, which may be catastrophic [9]. For instance, in latency-critical
IoT applications such as factory automation, smart grids, and intelligent transport systems [10]. Since the IoT devices usually have limited cache size, their data needs to be collected in time before it becomes obsolete or is overwritten by the new data. The authors in [6] proposed two UAV trajectory design algorithms, namely, the Max-AoI-optimal and Ave-AoIoptimal for effective data collection while considering the age of information (AoI) metric. In [7], the authors formulated an optimization problem to jointly optimize the UAV's trajectory, energy, and service time allocations to minimize the average Peak Age-of-information (PAoI) for a source-destination link. In [9], both the UAV trajectory and the service bandwidth allocation are optimized to maximize the total number of served ground IoT users, whereas the UAV is required to collect the devices' data within their delay requirements.

Unlike studies in [6]-[9], [11], [12] that only investigated the UL or DL channel and simply applied HD communication in their system. This is because the RT constraint should be considered as the latency from edge users (or GW) to GW (or edge users), wherein the $\mathrm{GW}$ is assumed to connect with a core network by stable connections, e.g., wired cable. The delay between the UAV and GW only can be neglected iff this value is very small. Motivated from the above discussions, this paper investigates the impact of latency constraints for both the UL and DL channels in UAV relay-assisted IoT systems. Especially, the UAV can be deployed in full-duplex (FD) or half-duplex (HD) mode for a comprehensive study of the advantages of each scheme. As the realistic requirement to improve the estimation accuracy in a natural disaster or emergency scenario, we propose to maximize the total collected throughput, subject to the maximum speed constraint of the UAV, total traveling time constant $T$, maximum transmit power at the devices/UAV, limited cache size of UAV, and latency constraints for both UL and DL. Towards an efficient solution, we first transform the relaxed problem into a computationally tractable form by introducing additional variables and adding an entropy-based penalty function. We then develop a lowcomplexity iterative algorithm by leveraging the well-known inner approximation (IA) method [13], which solves only a simple convex problem at each iteration. In order to assess the performance of the obtained solutions, extensive numerical results are provided to show the enhanced performance compared with the benchmarks. 


\section{$\mathrm{T}$}

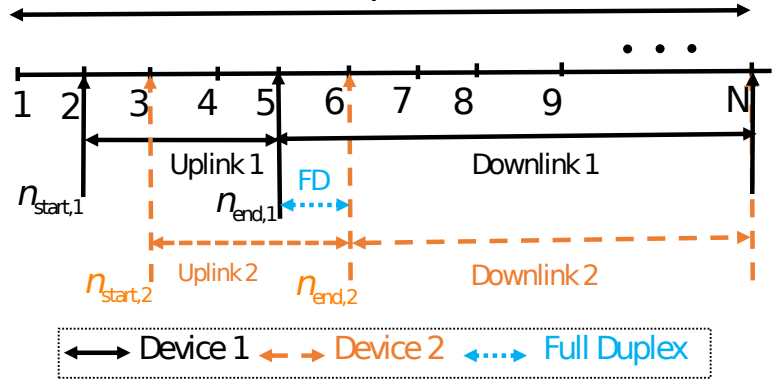

Fig. 1: Illustration of the data transmission process of 2 IoT devices with $N$ time intervals. The first IoT device with initial data transmission time at $n_{\text {start, } 1}=2$, timeout at $n_{\text {end,1 }}=5$. The second IoT device with initial data transmission time at $n_{\text {start }, 2}=3$, timeout at $n_{\mathrm{end}, 2}=6$. The UAV operates in the FD mode from time slots 5 to 6 since two devices utilize the same sub-carrier.

\section{System Model and Problem Formulation}

We consider a wireless communication system where a UAV acts as a relay to receive data from a set $\mathcal{K} \triangleq\{1, \ldots, K\}$ of $K$ IoT devices and then forward to a ground gateway (GW). Each IoT device is equipped with a single antenna and operates in the HD mode. The UAV is capable of adopting either FD or HD mode. The total flying time of UAV is limited by $T$. Each device is active at different time instances $t$, where $0 \leq t \leq T$. The device $k$ 's location are represented by $r_{k} \in \mathbb{R}^{2 \times 1}, k \in$ $\mathcal{K}$. We assume that the IoT locations, data sizes, the starting data transmission time (i.e., $n_{\mathrm{start}, k}$ ), and deadline time (i.e., $\left.n_{\text {end, } k}\right)$ are known to the UAV through the control center. As shown in Fig. 1, we denote $n_{\text {start }, k}$ and $n_{\mathrm{end}, k}$ as the starting data transmission time and latency constraint of the device $k$, respectively. It is assumed that the UAV must collect device $k$ 's data during period $n_{\text {start }, k} \leq t \leq n_{\text {end, } k}$. For simplicity, the UAV flies at fixed altitude $H$ (meters), which is the minimum altitude to avoid obstacles [5]. Denote by $q(t) \in \mathbb{R}^{2 \times 1}$ with $0 \leq$ $t \leq T$ the UAV trajectory projected onto the horizontal plane. For tractability, $T$ is divided into $N$ equally slots, i.e., $T=N \delta_{t}$ with $\delta_{t}$ denotes the duration of each time slot. Thus, $q(t)$ can be represented as $(q[n])_{n=1}^{N}$, where $q[n]$ is the UAV's horizontal location at $n$-th time slot. Let $V_{\max }$ denote the maximum flying speed of the UAV. We then have the UAV's speed constraint $\|q[n]-q[n-1]\| \leq \delta_{d}=V_{\max } \delta_{t}, n=2, \ldots, N$. Moreover, $\mathcal{N}=\{1, \ldots, N\}$ denotes the set of all time slots.

Let $k$ and $\mathrm{U}$ represent for the $k$-th IoT device and the UAV, respectively. Henceforth, $1 k$ and $2 k$ denote the channel $k \rightarrow$ $\mathrm{U}$ and $\mathrm{U} \rightarrow \mathrm{GW}$ to convey the data of user $k$, respectively. Then, the distance from $k \rightarrow \mathrm{U}$ or $\mathrm{U} \rightarrow \mathrm{GW}$ is $d_{i k}[n]=$ $\sqrt{H^{2}+\|q[n]-\mathbf{r}\|^{2}}, \forall n, k$, where $i \in\{1,2\}, \mathbf{r} \in\left\{r_{k}, q_{0}\right\}$, with $q_{0}$ denotes the GW's location.

This work considers a practical channel model including both large-scale and small-scale fading [14]. Concretely, the channel coefficient at $n$-th time slot, $h_{i k}[n]$, is decomposed as [9]

$$
h_{i k}[n]=\sqrt{\omega_{i k}[n]} \tilde{h}_{i k}[n],
$$

where $\omega_{i k}[n]=\omega_{0} d_{i k}^{-\alpha}[n]$ represents for the large-scale fading effects, with $\omega_{0}$ is the average channel power gain at the reference distance $d=1$ meter, and $\alpha$ is the path loss exponent. $\tilde{h}_{i k}[n]$ accounts for the Rician small-scale fading coefficient in the form of $\tilde{h}_{i k}[n]=\sqrt{\frac{G}{1+G}} \bar{h}_{i k}[n]+\sqrt{\frac{1}{1+G}} \hat{h}_{i k}[n]$. Here, $G$ is the Rician factor; $\bar{h}_{i k}[n]$ and $\hat{h}_{i k}[n] \sim \mathcal{C N}(0,1)$ denote the LoS and NLoS components, respectively.

The achievable transmission rate (bits/s) of links $k \rightarrow \mathrm{U}$ and $\mathrm{U} \rightarrow \mathrm{GW}$ to convey the data of device $k$ at time slot $n$, for the FD and HD operations, are respectively given by

$$
r_{1 k}[n]=\left\{\begin{array}{l}
r_{1 k}^{\mathrm{FD}}[n]=a_{1 k}[n] B \\
\log _{2}\left(1+\frac{\frac{p_{1 k}[n]\left|\tilde{h}_{1 k}[n]\right|^{2} \omega_{0}}{\left(H^{2}+\left\|q[n]-r_{k}\right\|^{2}\right)^{\alpha / 2}}}{\phi^{\mathrm{RSI}} \sum_{k^{\star} \in \mathcal{K} \backslash k}^{K} p_{2 k^{*}}[n]+\sigma^{2}}\right) \\
r_{2 k}[n] \stackrel{{ }_{1 k}^{\mathrm{HD}}[n]=a_{1 k}[n] B}{\log _{2}\left(1+\frac{p_{1 k}[n]\left|\tilde{h}_{1 k}[n]\right|^{2} \omega_{0}}{\left(H^{2}+\left\|q[n]-r_{k}\right\|^{2}\right)^{\alpha / 2} \sigma^{2}}\right)} \\
a_{2}[n] B \\
\log _{2}\left(1+\frac{p_{2 k}[n]\left|\tilde{h}_{2 k}[n]\right|^{2} \omega_{0}}{\left(H^{2}+\left\|q[n]-q_{0}\right\|^{2}\right)^{\alpha / 2} \sigma^{2}}\right)
\end{array}\right.
$$

where $\phi^{\mathrm{RSI}} \triangleq \rho^{\mathrm{RSI}}\left|g_{\mathrm{U}}[n]\right|^{2}$ with $\rho^{\mathrm{RSI}} \in[0,1)$ is the residual SI suppression (SiS) level after real-time cancellations in analogdigital domains and $g_{\mathrm{U}}[n]$ denotes the fading loop channel at UAV which interferes the UL reception due to the concurrent DL transmission; $\sigma^{2}$ denotes the power of additive white Gaussian noise (AWGN); $B$ is the total bandwidth in hertz $(\mathrm{Hz})$; $a_{1 k}[n] B$ and $a_{2 k}[n] B$ are the the portion of bandwidth allocated for the UL and DL to transmit data of $k$-th device during time slot $n$, respectively. In practice, for a large number of resources, $a_{1 k}[n]$ and $a_{2 k}[n]$ are approximately continuous between 0 and 1 . Thus, the bandwidth allocation should comply with the following constraints $\sum_{k \in \mathcal{K}} a_{i k}[n] \leq 1,0 \leq a_{i k}[n] \leq 1, \forall k, n$.

From (2)-(3), the throughput of UL and DL to transmit the data of device $k$ at time slot $n$ are

$$
\begin{aligned}
& C_{1 k}[n]=\delta_{t} R_{1 k}[n], \\
& C_{2 k}[n]=\delta_{t} R_{2 k}[n],
\end{aligned}
$$

respectively, where

$$
\begin{aligned}
& R_{1 k}[n]= \begin{cases}r_{1 k}[n], & \text { if } n \in \mathcal{T}_{1 k} \\
0, & \text { otherwise }\end{cases} \\
& R_{2 k}[n]= \begin{cases}r_{2 k}[n], & \text { if } n \in \mathcal{T}_{2 k} \\
0, & \text { otherwise }\end{cases}
\end{aligned}
$$

with $\mathcal{T}_{1 k} \triangleq\left\{n_{\text {start }, k}, \ldots, n_{\text {end }, k}\right\}, \mathcal{T}_{2 k} \triangleq\left\{n_{\text {end }, k}+1, \ldots, N\right\}$; (6) implies that the UL transmission $k \rightarrow \mathrm{U}$ only occurs during time period $\mathcal{T}_{1 k}$. On the other hand, the DL transmission $\mathrm{U} \rightarrow$ $\mathrm{GW}$ is conducted iff the UAV finishes the data collection for that device, as in (7). Moreover, the total achieved throughput 
on the $\mathrm{UL}$ and DL are represented as $C_{1 k} \triangleq \sum_{n \in \mathcal{T}_{1 k}} \delta_{t} R_{1 k}[n]$ and $C_{2 k} \triangleq \sum_{n \in \mathcal{T}_{2 k}} \delta_{t} R_{2 k}[n]$, respectively.

Then, we have the latency constraints

$$
\begin{aligned}
\lambda_{k} \frac{S_{k}}{R_{1 k}} & \leq\left(n_{\mathrm{end}, k}-n_{\mathrm{start}, k}\right) \delta_{t}, \forall k, \\
\lambda_{k} \frac{S_{k}}{R_{2 k}} & \leq\left(N-n_{\mathrm{end}, k}\right) \delta_{t}, \forall k,
\end{aligned}
$$

where $S_{k}$ denotes the data size, $R_{1 k} \triangleq \sum_{n \in \mathcal{T}_{1 k}} R_{1 k}[n], R_{2 k} \triangleq$ $\sum_{n \in \mathcal{T}_{2 k}} R_{2 k}[n]$; (8) signifies that the device $k$ must transmit the data to UAV before the latency constraint; (9) implies that the DL process is performed during the flying time of UAV. ${ }^{1}$

A practical operational constraint of the limited storage capacity of $C$ for the UAV is considered, which is stated as

$$
\sum_{k \in \mathcal{K}}\left(\sum_{l=1}^{n} C_{1 k}[l]-\sum_{l=1}^{n-1} C_{2 k}[l]\right) \leq C, \forall n,
$$

where $\sum_{l=1}^{n} C_{1 k}[l] \triangleq \lambda_{k} S_{k}-\sum_{l=n+1}^{N} \delta_{t} R_{1 k}[l], C_{2 k}[l] \triangleq \delta_{t} R_{2 k}[l]$. Notably, to spend a part of storage capacity for future use, i.e., a free cache size to store new incoming data, the amount of data stored at the UAV is calculated as the size of files collected from all devices till $(n)$-th time slot minus files transmitted to GW till $(n-1)$-th time slot as in (10).

To mathematically describe a successfully served device, a new binary variable $\lambda_{k}$ is introduced

$$
\lambda_{k}=\left\{\begin{array}{lr}
1, \text { Device } k \text { is successfully served } \\
0, & \text { otherwise. }
\end{array}\right.
$$

Let us define $\mathbf{q} \triangleq\{q[n], \forall n\}, \mathbf{a} \triangleq\left\{a_{1 k}[n], a_{2 k}[n], k \in\right.$ $\mathcal{K}, n \in \mathcal{N}\}, \mathbf{p} \triangleq\left\{p_{1 k}[n], p_{2 k}[n], k \in \mathcal{K}, n \in \mathcal{N}\right\}, \boldsymbol{\lambda} \triangleq$ $\left\{\lambda_{k}, k \in \mathcal{K}\right\}$. By taking into account the storage limitation, under the assumption that the locations, initial transmission time, and timeout constraints of all IoT devices are known a priori. Consequently, the problem for maximizing the total throughput by jointly optimizing the UAV trajectory, the allocation of resources (i.e., bandwidth and transmit power assigned

\footnotetext{
${ }^{1}$ We consider the system model in which the UAV does not transmit the data during taking off and landing [15]. Thus, the data transmission process only occurs when UAV is flying in the sky.
}

for UL/DL) can be formulated as

$$
\begin{array}{ll}
\mathcal{P}: & \max _{\mathbf{q}, \mathbf{a}, \mathbf{p}, \boldsymbol{\lambda}} \sum_{k \in \mathcal{K}} \delta_{t} \min \left(R_{1 k}, R_{2 k}\right) \\
\text { s.t. } & (8),(9),(10), \\
& \lambda_{k} \in\{0,1\}, \forall k, n, \\
& \delta_{t} \min \left(R_{1 k}, R_{2 k}\right) \geq \lambda_{k} S_{k}, \forall k, \\
& \sum_{k=1}^{K} \delta_{t} R_{2 k} \geq \sum_{k=1}^{K} \lambda_{k} S_{k}, \forall k, \\
& \sum_{k \in \mathcal{K}} \lambda_{k} \geq \lambda_{\text {thresh }}, \forall k \\
& \sum_{k \in \mathcal{K}} a_{i k}[n] \leq 1, \forall n \\
& 0 \leq a_{i k}[n] \leq 1, \forall k, n . \\
& q[1]=q_{I}, q[N]=q_{F}, \\
& \|q[n]-q[n-1]\| \leq \delta_{d}, n=2, \ldots, N, \\
& 0 \leq p_{1 k}[n] \leq P_{k}^{\max }[n], \forall k, n, \\
& 0 \leq \sum_{k=1}^{K} p_{2 k}[n] \leq P_{\mathrm{U}}^{\max }[n], \forall n,
\end{array}
$$

where constraint (12d) means that each served IoT device uploads the minimum amount of data $S_{k}$. Constraint (12e) guarantees that the device $k$ is successfully served iff all the data uploaded on the UL is transmitted on the DL. Constraint (12f) points out that the total number of served IoT devices must be larger than a given threshold value. Constraint (12i) takes care of the initial and final locations of the UAV. Constraints (12k) and (121) show that the total transmit power of device $k$ and the UAV is lower than or equal to their maximum power budgets $P_{k}^{\max }[n]$ and $P_{\mathrm{U}}^{\max }[n]$, respectively.

Analytically, it is evident that problem $\mathcal{P}$ is a mixed-integer non-linear program (MINLP), which is generally NP-hard. In addition, the binary constraint $(12 \mathrm{c})$ and the non-convex constraints (12b), (12d), and (12e) introduces intractability. There is no standard method for solving such a problem efficiently. Nevertheless, at least a locally optimal solution may be obtained by employing adequate relaxations to $\mathcal{P}$. In the sequel, we introduce an approximation method for $\mathcal{P}$, followed by its corresponding solution.

\section{Proposed Iterative Algorithm for Solving $\mathcal{P}$}

This section provides an iterative algorithm based on the IA method to solve the design problem.

\section{A. Tractable Formulation for $\mathcal{P}$}

To bypass the difficulty of binary nature of $\mathcal{P}$, we relax binary variables to continues ones as $0 \leq \lambda_{k} \leq$ $1, \forall k$. Then, we respectively introduce slack variables $z_{1 k}[n]$, $z_{2 k}[n]$, and $t_{1 k}[n]$ such that $\left(H^{2}+\left\|q[n]-r_{k}\right\|^{2}\right) \leq$ $\left(z_{1 k}[n]\right)^{2 / \alpha}, \quad\left(H^{2}+\left\|q[n]-q_{0}\right\|^{2}\right) \leq\left(z_{2 k}[n]\right)^{2 / \alpha}, \quad$ and 
$\left(\phi^{\mathrm{RSI}} \sum_{k^{\star} \in \mathcal{K} \backslash k} p_{2 k^{*}}[n]+\sigma^{2}\right) \leq t_{1 k}[n]$, which yields

$$
\begin{aligned}
& r_{1 k}[n] \geq\left\{\begin{array}{l}
r_{1 k}^{\mathrm{FD}, \mathrm{lb}}[n]=a_{1 k}[n] B \\
\log _{2}\left(1+\frac{p_{1 k}[n]\left|\tilde{h}_{1 k}[n]\right|^{2} \omega_{0}}{z_{1 k}[n] t_{1 k}[n]}\right), \\
r_{1 k}^{\mathrm{HD}, \mathrm{lb}}[n]=a_{1 k}[n] B \\
\log _{2}\left(1+\frac{p_{1 k}[n]\left|\tilde{h}_{1 k}[n]\right|^{2} \omega_{0}}{z_{1 k}[n] \sigma^{2}}\right),
\end{array}\right. \\
& r_{2 k}[n]=a_{2 k}[n] B \\
& \log _{2}\left(1+\frac{p_{2 k}[n]\left|\tilde{h}_{2 k}[n]\right|^{2} \omega_{0}}{z_{2 k}[n] \sigma^{2}}\right) .
\end{aligned}
$$

By substituting (13) and (14) into (4), (5), (6), and (7), we obtain $C_{1 k}^{\mathrm{lb}}[n], C_{2 k}^{\mathrm{lb}}[n], R_{1 k}^{\mathrm{lb}}[n]$, and $R_{2 k}^{\mathrm{lb}}[n]$, respectively. Moreover, we have $R_{1 k}^{\mathrm{lb}} \triangleq \sum_{n \in \mathcal{T}_{1 k}}^{1} R_{1 k}^{\mathrm{lb}}[n], R_{2 k}^{\mathrm{lb}} \triangleq \sum_{n \in \mathcal{T}_{2 k}} R_{2 k}[n]$. Next, the total throughput over $N$ time slots received on the $\mathrm{UL}$ and $\mathrm{DL}$ can be rewritten as $C_{1 k}^{\mathrm{lb}} \triangleq \sum_{n \in \mathcal{T}_{1 k}} \delta_{t} R_{1 k}[n]$ and $C_{2 k}^{\mathrm{lb}} \triangleq \sum_{n \in \mathcal{T}_{2 k}} \delta_{t} R_{2 k}[n]$, respectively. Let us denote $\mathbf{z} \triangleq$ $\left\{z_{1 k}[n], z_{2 k}[n], n \in \mathcal{N}, k \in \mathcal{K}\right\}, \mathbf{t} \triangleq\left\{t_{1 k}[n], k \in \mathcal{K}, n \in \mathcal{N}\right\}$. Then, the problem $\mathcal{P}$ can be re-written as

$$
\begin{aligned}
& \mathcal{P}_{\text {relaxed }}: \max _{\mathbf{q}, \mathbf{a}, \mathbf{p}, \boldsymbol{\lambda}, \mathbf{z}, \mathbf{t}} \sum_{k \in \mathcal{K}} \delta_{t} \min \left(R_{1 k}^{\mathrm{lb}}, R_{2 k}^{\mathrm{lb}}\right) \\
& \text { s.t. }(12 \mathrm{f}),(12 \mathrm{~g}),(12 \mathrm{~h}),(12 \mathrm{i}),(12 \mathrm{j}),(12 \mathrm{k}),(12 \mathrm{l}) \text {, } \\
& 0 \leq \lambda_{k} \leq 1, \forall k \\
& \left(H^{2}+\left\|q[n]-r_{k}\right\|^{2}\right) \leq\left(z_{1 k}[n]\right)^{2 / \alpha}, \forall k, n, \\
& \left(H^{2}+\left\|q[n]-q_{0}\right\|^{2}\right) \leq\left(z_{2 k}[n]\right)^{2 / \alpha}, \forall n, \\
& \left(\phi^{\mathrm{RSI}} \sum_{k^{*}=1, k^{*} \neq k}^{K} p_{2 k^{*}}[n]+\sigma^{2}\right) \leq t_{1 k}[n], \forall k, n,( \\
& \lambda_{k} \frac{S_{k}}{R_{1 k}^{\mathrm{lb}}} \leq\left(n_{\mathrm{end}, k}-n_{\mathrm{start}, k}\right) \delta_{t}, \forall k, \\
& \lambda_{k} \frac{S_{k}}{R_{2 k}^{\mathrm{lb}}} \leq\left(N-n_{\mathrm{end}, k}\right) \delta_{t}, \forall k, \\
& \delta_{t} \min \left(R_{1 k}^{\mathrm{lb}}, R_{2 k}^{\mathrm{lb}}\right) \geq \lambda_{k} S_{k}, \forall k, \\
& \sum_{k=1}^{K} \delta_{t} R_{2 k}^{\mathrm{lb}} \geq \sum_{k=1}^{K} \lambda_{k} S_{k}, \forall k \in \mathcal{K} \\
& \sum_{k \in \mathcal{K}}\left(\lambda_{k} S_{k}-\sum_{l=n+1}^{N} \delta_{t} R_{1 k}^{\mathrm{lb}}[l]-\sum_{l=1}^{n-1} \delta_{t} R_{2 k}^{\mathrm{lb}}[l]\right) \leq C,
\end{aligned}
$$

$\forall k, n$.

Although problem $\mathcal{P}_{\text {relaxed }}$ is still non-convex due to the non-convexity of constraints (15c)-(15j). In what follows, we apply IA framework to develop newly approximated functions to convexify non-convex parts.

\section{B. Proposed IA-based Algorithm}

We first treat the non-convex functions $r_{1 k}^{\mathrm{lb}}[n]$ and $r_{2 k}^{\mathrm{lb}}[n]$ in constraints (15f), (15g), (15h), (15i), and (15j). Specifically, they can be expressed as

$$
\begin{aligned}
& r_{1 k}^{\mathrm{lb}}[n] \triangleq a_{1 k}[n] \Phi_{1 k}[n] \geq a_{1 k}[n] \bar{\Phi}_{1 k}[n], \\
& r_{2 k}^{\mathrm{lb}}[n] \triangleq a_{2 k}[n] \Phi_{2 k}[n] \geq a_{2 k}[n] \bar{\Phi}_{2 k}[n],
\end{aligned}
$$

where

$$
\begin{aligned}
& \Phi_{1 k}[n] \triangleq B \log _{2}\left(1+\frac{p_{1 k}[n]\left|\tilde{h}_{1 k}[n]\right|^{2} \omega_{0}}{z_{1 k}[n] t_{1 k}[n]}\right), \\
& \Phi_{2 k}[n] \triangleq B \log _{2}\left(1+\frac{p_{2 k}[n]\left|\tilde{h}_{2 k}[n]\right|^{2} \omega_{0}}{z_{2 k}[n] \sigma^{2}}\right),
\end{aligned}
$$

$$
\bar{\Phi}_{1 k}[n] \triangleq B\left(\zeta_{1}+\zeta_{2}\left(p_{1 k}[n]\right)-\zeta_{3}\left(p_{1 k}[n], t_{1 k}[n], z_{1 k}[n]\right)\right) \text {, }
$$

$$
\bar{\Phi}_{2 k}[n] \triangleq B\left(\xi_{1}+\xi_{2}\left(p_{2 k}[n]\right)-\xi_{3}\left(p_{2 k}[n], z_{2 k}[n]\right)\right) .
$$

Proof: See Appendix A.

By introducing the slack variables $\Phi_{1 k}^{\mathrm{lb}}[n]$ and $\Phi_{1 k}^{\mathrm{lb}}[n]$, we have $\bar{\Phi}_{1 k}[n] \geq \Phi_{1 k}^{\mathrm{lb}}[n], \bar{\Phi}_{2 k}[n] \geq \Phi_{2 k}^{\mathrm{lb}}[n]$. Consequently, $r_{1 k}^{\mathrm{lb}}[n]$ and $r_{2 k}^{\mathrm{lb}}[n]$ are converted to the following constraints:

$$
\begin{aligned}
& r_{1 k}^{\mathrm{lb}}[n] \geq \bar{r}_{1 k}^{\mathrm{lb}}[n] \triangleq a_{1 k}[n] \Phi_{1 k}^{\mathrm{lb}}[n], \\
& r_{2 k}^{\mathrm{lb}}[n] \geq \bar{r}_{2 k}^{\mathrm{lb}}[n] \triangleq a_{2 k}[n] \Phi_{2 k}^{\mathrm{lb}}[n] .
\end{aligned}
$$

To deal with the non-convexity of right hand sides (RHSs) of (22) and (23), we replace $a_{1 k}[n] \Phi_{1 k}^{\mathrm{lb}}[n]$ and $a_{2 k}[n] \Phi_{2 k}^{\mathrm{lb}}[n]$ by $0.25\left[\left(a_{1 k}[n]+\Phi_{1 k}^{\mathrm{lb}}[n]\right)^{2}-\left(a_{1 k}[n]-\Phi_{1 k}^{\mathrm{lb}}[n]\right)^{2}\right]$ and $0.25\left[\left(a_{2 k}[n]+\Phi_{2 k}^{\mathrm{lb}}[n]\right)^{2}-\left(a_{2 k}[n]-\Phi_{2 k}^{\mathrm{lb}}[n]\right)^{2}\right]$, respectively. Then, we apply the first-order Taylor to approximate the convex functions $\left(a_{1 k}[n]+\Phi_{1 k}[n]\right)^{2}$ and $\left(a_{2 k}[n]+\Phi_{2 k}[n]\right)^{2}$ at $(j+1)$-th iteration, respectively. Thus, we have

$$
\begin{aligned}
a_{1 k}[n] \Phi_{1 k}^{\mathrm{lb}}[n] & \geq \frac{\left(a_{1 k}^{(j)}[n]+\Phi_{1 k}^{\mathrm{lb},(j)}[n]\right)^{2}}{4}+\frac{\left(a_{1 k}^{(j)}[n]+\Phi_{1 k}^{\mathrm{lb},(j)}[n]\right)}{2} \\
& \times\left(a_{1 k}[n]-a_{1 k}^{(j)}[n]+\Phi_{1 k}^{\mathrm{lb}}[n]-\Phi_{1 k}^{\mathrm{lb},(j)}[n]\right) \\
& -\frac{\left(a_{1 k}[n]-\Phi_{1 k}^{\mathrm{lb}}[n]\right)^{2}}{4} \triangleq \tilde{r}_{1 k}^{\mathrm{lb}}[n], \\
a_{2 k}[n] \Phi_{2 k}^{\mathrm{lb}}[n] & \geq \frac{\left(a_{2 k}^{(j)}[n]+\Phi_{2 k}^{\mathrm{lb},(j)}[n]\right)^{2}}{4}+\frac{\left(a_{2 k}^{(j)}[n]+\Phi_{1 k}^{\mathrm{lb},(j)}[n]\right)}{2} \\
& \times\left(a_{2 k}[n]-a_{2 k}^{(j)}[n]+\Phi_{2 k}^{\mathrm{lb}}[n]-\Phi_{2 k}^{\mathrm{lb},(j)}[n]\right) \\
& -\frac{\left(a_{2 k}[n]-\Phi_{2 k}^{\mathrm{lb}}[n]\right)^{2}}{4} \triangleq \tilde{r}_{2 k}^{\mathrm{lb}}[n] .
\end{aligned}
$$

To convexify (15e)-(15j), we introduce slack variables $\widehat{r}_{1 k}^{\mathrm{lb}}[n]$ and $\widehat{r}_{2 k}^{\mathrm{b}}[n]$, such that

$$
\begin{aligned}
& \tilde{r}_{1 k}^{\mathrm{lb}}[n] \geq \widehat{r}_{1 k}^{\mathrm{bb}}[n], \\
& \tilde{r}_{2 k}^{\mathrm{lb}}[n] \geq \widehat{r}_{2 k}^{\mathrm{bb}}[n] .
\end{aligned}
$$

Consequently, substituting $\widehat{r}_{1 k}^{\mathrm{bb}}[n]$ and $\widehat{r}_{2 k}^{\mathrm{b}}[n]$ into (4)-(7), we obtain $\widehat{C}_{1 k}^{\mathrm{lb}}[n], \widehat{C}_{2 k}^{\mathrm{lb}}[n], \widehat{R}_{1 k}^{\mathrm{lb}}[n]$, and $\widehat{R}_{2 k}^{\mathrm{lb}}[n]$, respectively. 


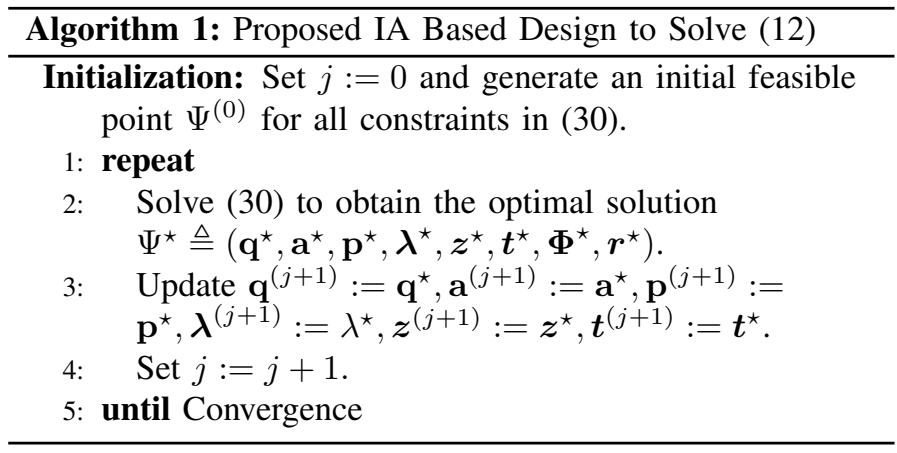

Moreover, we have $\widehat{R}_{1 k}^{\mathrm{lb}} \triangleq \sum_{n \in \mathcal{T}_{1 k}} \widehat{R}_{1 k}^{\mathrm{lb}}[n], \widehat{R}_{2 k}^{\mathrm{lb}} \triangleq \sum_{n \in \mathcal{T}_{2 k}} \widehat{R}_{2 k}^{\mathrm{lb}}[n]$. Next, the total throughput over $N$ time slots received on the UL and DL can be rewritten as $\widehat{C}_{1 k}^{\mathrm{lb}} \triangleq \sum_{n \in \mathcal{T}_{1 k}} \delta_{t} \widehat{R}_{1 k}^{\mathrm{lb}}[n]$ and $\widehat{C}_{2 k}^{\mathrm{lb}} \triangleq \sum_{n \in \mathcal{T}_{2 k}} \delta_{t} \widehat{R}_{2 k}^{\mathrm{lb}}[n]$, respectively. Particularly, as the binary variable $\lambda_{k}$ is relaxed to continuous values between 0 and 1 (15c) which does not guarantee that $\lambda_{k}$ converges to 0 or 1 . This motivates us to execute a penalty function $\mathbb{P}\left(\lambda_{k}\right) \triangleq \lambda_{k} \ln \left(\lambda_{k}\right)+\left(1-\lambda_{k}\right) \ln \left(1-\lambda_{k}\right)$ [16]. Since $\mathbb{P}\left(\lambda_{k}\right)$ is convex for $\lambda_{k} \geq 0$, we replace $\mathbb{P}\left(\lambda_{k}\right)$ by its first order Taylor approximation at $(j+1)$-th iteration:

$$
\widehat{\mathbb{P}}\left(\lambda_{k}\right) \triangleq \kappa\left(\mathbb{P}\left(\lambda_{k}^{(j)}\right)+\nabla \mathbb{P}\left(\lambda_{k}^{(j)}\right)\left(\lambda_{k}-\lambda_{k}^{(j)}\right)\right)
$$

where

$$
\nabla \mathbb{P}\left(\lambda_{k}^{(j)}\right)=\ln \left(\lambda_{k}^{(j)}\right)-\ln \left(1-\lambda_{k}^{(j)}\right)
$$

Let us denote $\boldsymbol{\Phi} \triangleq\left\{\Phi_{1 k}^{\mathrm{lb}}[n], \Phi_{2 k}^{\mathrm{lb}}[n], \forall k, n\right\}$ and $\mathbf{r} \triangleq$ $\left\{\widehat{r}_{1 k}^{\mathrm{b}}[n], \widehat{r}_{2 k}^{\mathrm{lb}}[n], \forall k, n\right\}$. Bearing all the above developments in mind, we solve the following approximate convex program at $(j+1)$-th iteration:

$$
\begin{aligned}
\mathcal{P}_{\text {convex }} & \max _{\mathbf{q}, \mathbf{a}, \mathbf{p}, \boldsymbol{\lambda}, \boldsymbol{z}, \boldsymbol{t}, \mathbf{\Phi}, \mathbf{r},} \sum_{k \in \mathcal{K}} \delta_{t} \min \left(\widehat{R}_{1 k}, \widehat{R}_{2 k}\right) \\
\text { s.t. } & (15 \mathrm{~b}),(15 \mathrm{c}),(15 \mathrm{~d}),(15 \mathrm{e}),(26),(27), \\
& \lambda_{k} \frac{S_{k}}{\widehat{R}_{1 k}^{\mathrm{lb}}} \leq\left(n_{\mathrm{end}, k}-n_{\mathrm{start}, k}\right) \delta_{t}, \forall k \\
& \lambda_{k} \frac{S_{k}}{\widehat{R}_{2 k}^{\mathrm{lb}}} \leq\left(N-n_{\mathrm{end}, k}\right) \delta_{t}, \forall k \\
& \delta_{t} \min \left(\widehat{R}_{1 k}^{\mathrm{lb}}, \widehat{R}_{2 k}^{\mathrm{lb}}\right) \geq \lambda_{k} S_{k}, \forall k \\
& \sum_{k=1}^{K} \delta_{t} \widehat{R}_{2 k}^{\mathrm{lb}} \geq \sum_{k=1}^{K} \lambda_{k} S_{k}, \\
& \sum_{k \in \mathcal{K}}\left(\lambda_{k} S_{k}-\sum_{l=n+1}^{N} \delta_{t} \widehat{R}_{1 k}^{\mathrm{lb}}[l]-\sum_{l=1}^{n-1} \delta_{t} \widehat{R}_{2 k}^{\mathrm{lb}}[l]\right) \leq C, \\
& \forall k, n,
\end{aligned}
$$

where $\widehat{R}_{1 k} \triangleq \widehat{R}_{1 k}^{\mathrm{lb}}+\kappa \lambda_{k} \nabla \mathbb{P}\left(\lambda_{k}^{(j)}\right), \widehat{R}_{2 k} \triangleq \widehat{R}_{2 k}^{\mathrm{lb}}+\kappa \lambda_{k} \nabla \mathbb{P}\left(\lambda_{k}^{(j)}\right)$. We successively solve the convex program (30) and update the involved optimization variables until convergence, which output at least a locally optimal solution to the relaxed problem. The detailed algorithm is summarized in Algorithm 1.

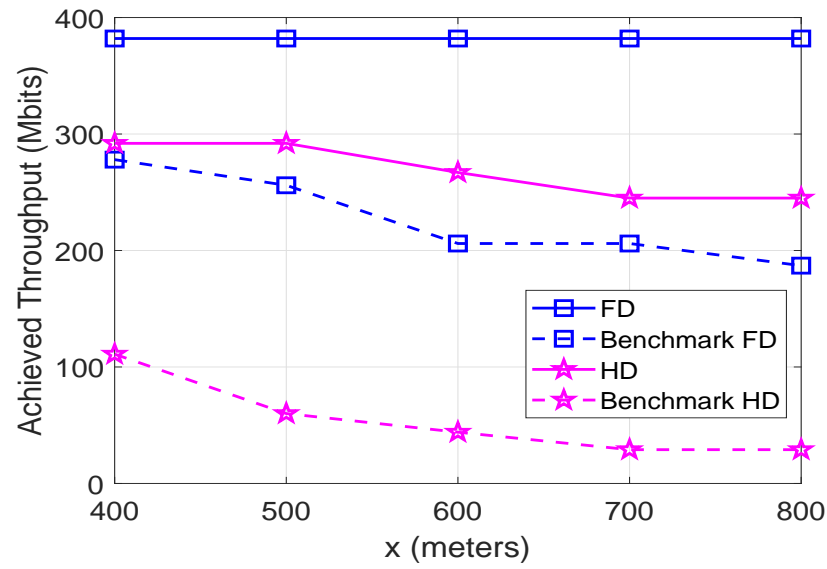

(a) $B=10$

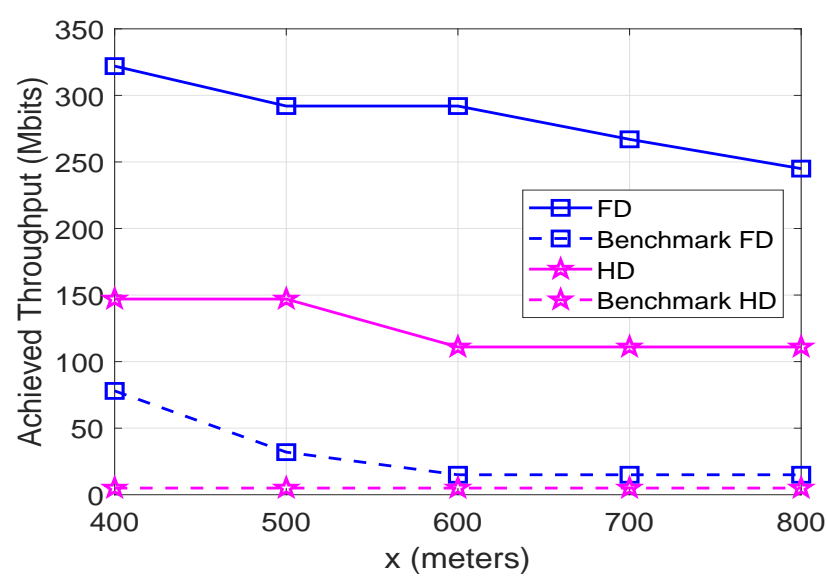

(b) $B=5$

Fig. 2: Total achievable throughput vs. different network sizes.

\section{NuMERICAL RESUlTS}

In this section, we perform the numerical evaluations to validate the proposed designs. Specifically, we consider $K$ IoT devices randomly distributed within the considered area, i.e., $500 \mathrm{~m} \times 500 \mathrm{~m}$, and the ground gateway is located at $(0,500 \mathrm{~m})$. The parameters are set as follows: $K=20$, $H=100$ meters, $B=10 \mathrm{MHz}$, path loss exponent $\alpha=2.3$, $\sigma^{2}=-110 \mathrm{dBm}, \omega_{0}=-40 \mathrm{~dB}, P_{U}^{\max }=20 \mathrm{dBm}, P_{k}^{\max }=$ $15 \mathrm{dBm}, S_{k}=30$ Mbits, Rician factor $\mathrm{G}=12 \mathrm{~dB}$, the maximum collection time deadline for each device $k n_{\mathrm{end}, k}$ is uniformly distributed between $n_{\mathrm{end}, k}^{\min }$ and $n_{\mathrm{end}, k}^{\max }$. The UAV's initial and final locations are deployed at $q_{I}=[300 \mathrm{~m}, 200 \mathrm{~m}]$ and $q_{F}=[100 \mathrm{~m}, 0]$, respectively. For comparison purpose, two benchmark schemes are considered. More specifically, the Benchmark FD and Benchmark HD are implemented similar to Algorithm 1 with the equal bandwidth allocated to each user, i.e., $a_{1 k}[n]=a_{1 k}[n]=\frac{B}{K}$.

In Fig. 2, we evaluate the total collected data (in Mbits) versus network sizes i.e., Area $=x^{2}$. It is observed that the larger the network size, the lower the throughput can be achieved. This is because IoT devices are distributed in 


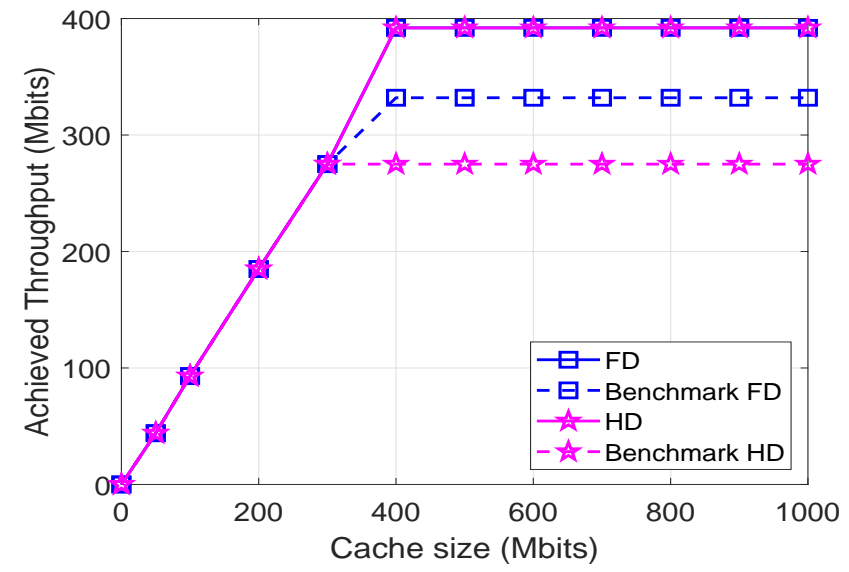

(a) $N=70$

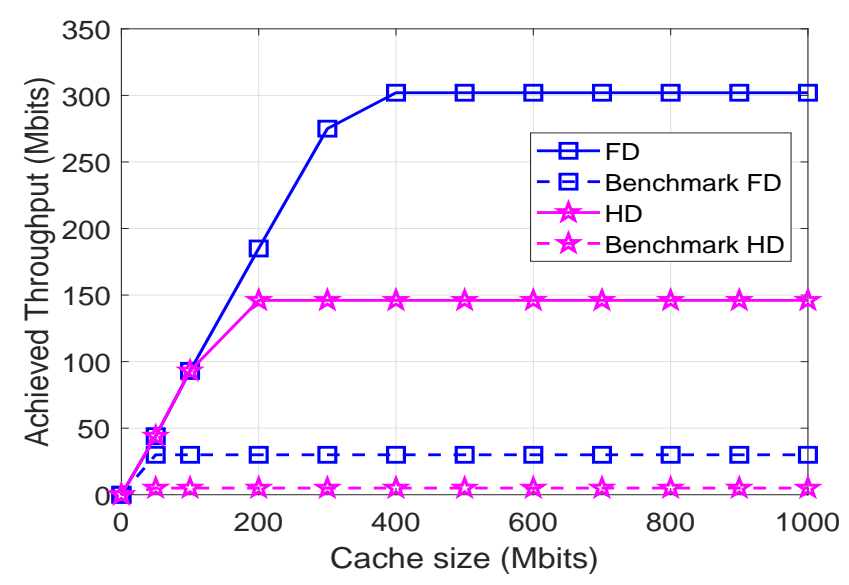

(b) $N=60$

Fig. 3: Total achievable throughput vs. different cache sizes.

a wider area. Thus, the UAV must fly at a higher speed to satisfy the time requirement of each device which is in contradiction with the $V_{\max }$ constraint. Moreover, the FD and HD methods outperform the benchmark ones due to the benefits of optimizing bandwidth allocation.

Fig. 3 studies the influence of total traveling time and storage capacity to the total amount of data, with $S_{k}$ is ranging from 5 to 30 Mbits. It is shown that the proposed algorithms significantly improve the performance compared with the references for all values of cache size (Mbits). Specifically, the FD algorithm can serve $77.04 \%$ of the total data amount and the HD algorithm achieves less than $39.8 \%$ at $C=400$ Mbits and $N=60$ time slots. Whereas the Benchmark FD and Benchmark HD respectively impose $7.65 \%$ and $1.28 \%$. Moreover, it is also observed that the more the traveling time is assigned, the higher the amount of data can be collected. Since the UAV has more time to exchange data between the IoT devices/GW. Particularly, at $N=70$, the HD scheme can obtain the same performance as the FD one since the UAV has enough time for collecting data from IoT users before transmitting to the GW. Hence, when the total traveling time is sufficiently large, the UAV should operate at HD mode for a simpler implementation in comparison to that of FD mode.

\section{CONClusion}

This work investigated the trajectory design for FD/HD UAVassisted IoT communication networks under latency constraints. Specifically, the total achieved throughput was maximized via jointly optimizing the UAV trajectory, allocated bandwidth, as well as the transmission power for the devices/UAV while complying with delay-sensitive data collection and limited storage capacity. Since our formulated problem was a mixed-integer non-convex program, we transformed the original problem into a convex form which was solved by an IA-based iterative algorithm. Finally, numerical results showed that our proposed designs outperform benchmark approaches.

\section{ACKNOWLEDGEMENT}

This research is supported by the Luxembourg National Research Fund under project FNR CORE ProCAST, grant C17/IS/11691338 and FNR CORE 5G-Sky, grant C19/IS/13713801.

\section{APPENDIX A}

From [17, Appendix B], [18], the upper bound of $z_{k}[n] t_{1 k}[n]$ in $r_{1 k}^{\mathrm{lb}}[n]$ is:

$$
\begin{aligned}
& z_{k}[n] t_{1 k}[n] \leq \\
& \left(z_{1 k}[n] t_{1 k}[n]\right)^{\mathrm{ub}} \triangleq \frac{z_{1 k}^{(j)}[n]\left(t_{1 k}[n]\right)^{2}}{2 t_{1 k}^{(j)}[n]}+\frac{t_{1 k}^{(j)}[n]\left(z_{1 k}[n]\right)^{2}}{2 z_{1 k}^{(j)}[n]},
\end{aligned}
$$

By using [19, Eq. (20)], and (A.1), the lower bounds of $\Phi_{1 k}[n]$ and $\Phi_{2 k}[n]$ are, respectively

$$
\begin{aligned}
\Phi_{1 k}[n] & \geq \bar{\Phi}_{1 k}[n] \\
& \triangleq B\left(\zeta_{1}+\zeta_{2}\left(p_{1 k}[n]\right)-\zeta_{3}\left(p_{1 k}[n], t_{1 k}[n], z_{1 k}[n]\right)\right),
\end{aligned}
$$

$$
\begin{aligned}
\Phi_{2 k}[n] & \geq \bar{\Phi}_{2 k}[n] \\
& \triangleq B\left(\xi_{1}+\xi_{2}\left(p_{2 k}[n]\right)-\xi_{3}\left(p_{2 k}[n], z_{2 k}[n]\right)\right),
\end{aligned}
$$

where the expressions corresponding to $\zeta_{1}, \zeta_{2}, \zeta_{3}, \xi_{1}, \xi_{2}$, and $\xi_{3}$ are provided in Table II.

\section{REFERENCES}

[1] Q. Pham, F. Fang, N. Ha, M. Le, Z. Ding, L. Le, and W. Hwang, "A survey of multi-access edge computing in $5 \mathrm{G}$ and beyond: Fundamentals, technology integration, and state-of-the-art," IEEE Access, 2019.

[2] S. Gautam, K. S. Shree, T. D. Hieu, S. Chatzinotas, and B. Ottersten, "Hybrid backscatter and relaying scheme for $6 \mathrm{~g}$ greencom iot networks with swipt," 10.36227/techrxiv.12893750.v1, 2020.

[3] K. Rose, S. Eldridge, and L. Chapin, "The internet of things: An overview," The Internet Society (ISOC), vol. 80, pp. 1-50, 2015.

[4] J. W. et al., "Energy-efficient data collection and device positioning in UAV-assisted IoT," IEEE Internet of Things J., vol. 7, no. 2, pp. 11221139, 2019.

[5] Y. Zeng, Q. Wu, and R. Zhang, "Accessing from the sky: A tutorial on UAV communications for 5G and beyond," Proc. of IEEE, vol. 107, no. 12 , pp. $2327-2375,2019$. 


\begin{tabular}{||l|l||}
\hline$\zeta_{1} \triangleq \log _{2}\left(1+\frac{p_{1 k}^{(j)}[n]\left|\tilde{h}_{1 k}[n]\right|^{2} \omega_{0}}{z_{1 k}^{(j)}[n] t_{1 k}^{(j)}[n]}\right)-\frac{p_{1 k}^{(j)}[n]\left|\tilde{h}_{1 k}[n]\right|^{2} \omega_{0}}{z_{1 k}^{(j)}[n] t_{1 k}^{(j)}[n] \ln 2}$, & $\xi_{1} \triangleq \log _{2}\left(1+\frac{p_{2 k}^{(j)}[n]\left|\tilde{h}_{2 k}[n]\right|^{2} \omega_{0}}{z_{2 k}^{(j)}[n] \sigma^{2}}\right)-\frac{p_{2 k}^{(j)}[n]\left|\tilde{h}_{2 k}[n]\right|^{2} \omega_{0}}{z_{2 k}^{(j)}[n] \sigma^{2} \ln 2}$, \\
\hline$\zeta_{2}\left(p_{1 k}[n]\right) \triangleq\left|\tilde{h}_{1 k}[n]\right|^{2} \omega_{0} \frac{2 \sqrt{p_{1 k}^{(j)}[n]} \sqrt{p_{1 k}[n]}}{z_{1 k}^{(j)}[n] t_{1 k}^{(j)}[n] \ln 2}$, & $\xi_{2}\left(p_{2 k}[n]\right) \triangleq \frac{\left|\tilde{h}_{2 k}[n]\right|^{2} \omega_{0}}{z_{2 k}^{(j)}[n] \sigma^{2} \ln 2} 2 \sqrt{p_{2 k}^{(j)}[n]} \sqrt{p_{2 k}[n]}$, \\
\hline$\zeta_{3}\left(p_{1 k}[n], t_{1 k}[n], z_{1 k}[n]\right) \triangleq \frac{1}{p_{1 k}^{(j)}[n]\left|\tilde{h}_{1 k}[n]\right|^{2} \omega_{0}+z_{1 k}^{(j)}[n] t_{1 k}^{(j)}[n]} \times \frac{p_{1 k}^{(j)}[n]\left|\tilde{h}_{1 k}[n]\right|^{2} \omega_{0}}{z_{1 k}^{(j)}[n] t_{1 k}^{(j)}[n] \ln 2}\left(p_{1 k}[n]\left|\tilde{h}_{1 k}[n]\right|^{2} \omega_{0}+\frac{z_{1 k}^{(j)}[n]\left(t_{1 k}[n]\right)^{2}}{2 t_{1 k}^{(j)}[n]}+\frac{t_{1 k}^{(j)}[n]\left(z_{1 k}[n]\right)^{2}}{2 z_{1 k}^{(j)}[n]}\right)$, \\
\hline$\xi_{3}\left(p_{2 k}[n], z_{2 k}[n]\right) \triangleq \frac{p_{2 k}^{(j)}[n]\left|\tilde{h}_{2 k}[n]\right|^{2} \omega_{0}}{p_{2 k}^{(j)}[n]\left|\tilde{h}_{2 k}[n]\right|^{2} \omega_{0}+z_{2 k}^{(j)}[n] \sigma^{2}} \times \frac{\left(p_{2 k}[n]\left|\tilde{h}_{2 k}[n]\right|^{2} \omega_{0}+z_{2 k}[n] \sigma^{2}\right)}{z_{2 k}^{(j)}[n] \sigma^{2} \ln 2}$ \\
\hline
\end{tabular}

TABLE I: Equations corresponding to Appendix A.

[6] J. Liu, X. Wang, B. Bai, and H. Dai, "Age-optimal trajectory planning for UAV-assisted data collection," in IEEE Conf. Computer Commun. Works. (INFOCOM WKSHPS), 2018, pp. 553-558.

[7] M. Abd-Elmagid and H. Dhillon, "Average peak age-of-information minimization in UAV-assisted IoT networks," IEEE Trans. Veh. Technol., vol. 68, no. 2, pp. 2003-2008, 2018.

[8] W. Li, L. Wang, and A. Fei, "Minimizing packet expiration loss with path planning in UAV-assisted data sensing," IEEE Wirel. Commun. Lett., vol. 8, no. 6, pp. 1520-1523, 2019.

[9] M. Samir, S. Sharafeddine, C. Assi, M. Nguyen, and A. Ghrayeb, "UAV trajectory planning for data collection from time-constrained IoT devices," IEEE Trans. Wireless Commun., vol. 19, no. 1, pp. 34-46, Jan. 2020.

[10] P. Schulz, M. Matthe et al., "Latency critical IoT applications in 5G: Perspective on the design of radio interface and network architecture," IEEE Commun. Mag., vol. 55, no. 2, pp. 70-78, 2017.

[11] D. H. Tran, T. X. Vu, S. Chatzinotas, and B. Ottersten, "Energyefficient trajectory design for UAV-enabled wireless communications with latency constraints," in 2019 53rd Asilomar Conf. Signals, Systems, and Computers, 2019, pp. 347-352.

[12] D. H. Tran, T. X. Vu, S. Chatzinotas, S. Shahbazpanahi, and B. Ottersten, "Coarse trajectory design for energy minimization in UAV-enabled wireless communications with latency constraints," IEEE Trans. Veh. Technol., June 2020 .
[13] B. Marks, "A general inner approximation algorithm for nonconvex mathematical programs," Oper. Res., vol. 26, no. 4, pp. 681-683, 1978.

[14] Y. Yuan, L. Lei, T. X. Vu, S. Chatzinotas, S. Sun, and B. Ottersten, "Energy minimization in UAV-aided networks: actor-critic learning for constrained scheduling optimization," in Arxiv, preprint arXiv:2006.13610.

[15] U. Challita, W. Saad, and C. Bettstetter, "Interference management for cellular-connected UAVs: A deep reinforcement learning approach," IEEE Trans. Wireless Commun., vol. 18, no. 4, pp. 2125-2140, April 2019.

[16] B. Ashok, B. S. Mysore, S. Chatzinotas, and B. Ottersten, "A joint solution for scheduling and precoding in multiuser MISO downlink channels," IEEE Trans. Wireless Comm., vol. 19, no. 1, pp. 475-490, Jan. 2019.

[17] V. D. Nguyen, H. V. Nguyen, O. A. Dobre, and O. S. Shin, "A new design paradigm for secure full-duplex multiuser systems," IEEE J. Select. Areas Commun., vol. 36, no. 7, pp. 1480-1498, July 2018.

[18] A. Beck, A. Ben-Tal, and L. Tetruashvili, "A sequential parametric convex approximation method with applications to nonconvex truss topology design problems," J. Global Optim., vol. 47, no. 1, pp. 29-51, May 2010.

[19] V. D. Nguyen, T. Q. Duong, H. D. Tuan, O. S. Shin, and H. V. Poor., "Spectral and energy efficiencies in full-duplex wireless information and power transfer," IEEE Trans. Commun, vol. 65, no. 5, pp. 2220-2233, May 2017. 